\title{
Softwares Educacionais para o Ensino de Programação: Um Mapeamento Sistemático
}

\author{
Anderson S. Marcolino, Ellen Francine Barbosa \\ ${ }^{1}$ Instituto de Ciências Matemáticas e de Computação \\ Universidade de São Paulo (ICMC-USP) - Departamento de Sistemas de Computação \\ Caixa Postal 668 - 13560-970 - São Carlos - SP - Brasil \\ andersonmarcolino@usp.br, francinedicmc.usp.br
}

\begin{abstract}
Several difficulties in programming courses have been led to adoption on the new information and communication technologies (ICTs) and different learning modalities for both face-to-face and distance education. This study looks to identify the software applications that support the programming education through use of new ICTs, learning modalities, software development technologies and pointing also to the existent gaps. To achieve these goals a systematic literature mapping was conducted. The selected studies allowed a creation of a catalog of contributions which adopt different teaching strategies to mitigate problems faced in programming disciplines. The results also showed trends and gaps to be researched in the domain, such as low exploration of systemic software reuse in software applications development and the incipient use of mobile and television learning modalities.
\end{abstract}

Resumo. As diversas dificuldades em cursos de programação têm repercutido, tanto na adesão de novas tecnologias da informação e comunicação (TICs), como na adoção de diferentes modalidades de ensino presencial e à distância. Este artigo busca identificar os softwares e aplicações educacionais que apoiam o ensino de programação, no que tange o uso de novas TICs, modalidades de ensino e tecnologias de desenvolvimento, apontando também as lacunas existentes. Para esse fim, um mapeamento sistemático de literatura foi conduzido. De modo geral, os estudos recuperados permitiram a criação de um catálogo de contribuições que adotam diferentes estratégias de ensino na mitigação de problemas enfrentados nas disciplinas de programação. Os resultados permitiram, também, identificar tendências e lacunas a serem pesquisadas, como a pouca exploração de reutilização sistêmica no processo de desenvolvimento dos softwares e aplicações, e o uso ainda incipiente das modalidades de ensino móvel e televisivas.

\section{Introdução}

Diversas iniciativas têm sido conduzidas na busca pela melhoria do processo de ensino e aprendizagem em diferentes áreas, cursos e disciplinas. Na área de computação, por exemplo, tem-se observado a intensificação de esforços em disciplinas que envolvem aspectos teóricos e práticos de programação. Esses esforços são reflexo, sobretudo, da importância das disciplinas de programação nas grades curriculares de cursos como Ciência da Computação, Sistemas de Informação, Engenharia de Computação e afins, tanto no contexto nacional [SBC 2003] como no cenário internacional [IEEE and ACM 2005]. 
Entre as principais causas das limitações e problemas relacionados ao ensino e aprendizagem de programação, destacam-se [Sales and Dantas 2010, S. et al. 2012]: (i) alto grau de abstração requerido; (ii) metodologias tradicionais de ensino que visam à aprendizagem de conceitos dinâmicos utilizando abordagens e materiais essencialmente estáticos; (iii) existência de alunos com backgrounds diversificados, levando a ritmos de aprendizagem diferenciados; (iv) dificuldade de acompanhamento individualizado do aluno; (v) necessidade de um estudo mais baseado na prática e (vi) linguagens de programação com sintaxes complexas e carência de representações visuais de algoritmos, dificultando a adequada compreensão dos conceitos de programação envolvidos. Já em relação aos discentes, existe a dificuldade no uso do raciocínio lógico e em enxergar os problemas propostos como um todo, relacionando-os com os conhecimentos de programação [Sales and Dantas 2010, S. et al. 2012, Mota et al. 2009]. Logo, tais aspectos acabam desmotivando alunos e professores e, por consequência, elevando os índices de evasão e reprova em disciplinas de programação.

Neste cenário, [Aureliano and Tedesco 2012] conduziram uma revisão sistemática com o objetivo de identificar as propostas de softwares para o ensino de programação para iniciantes [Aureliano and Tedesco 2012]. Os resultados apresentaram softwares desenvolvidos de modo singular e sem a adesão sistemática de métodos de reuso.

Este trabalho, assim como a revisão sistemática de [Aureliano and Tedesco 2012], tem como objetivo descrever um mapeamento sistemático para identificar os softwares e aplicações educacionais existentes na literatura. No entanto, tais trabalhos são investigados de modo geral, em nível nacional e internacional, não restringindo-se aos softwares e aplicações utilizados apenas no ensino de programação a iniciantes. Além disso, aspectos técnicos e estratégicos dos trabalhos também são explorados. As principais motivações para a condução deste trabalho estão relacionadas à criação de um catálogo de contribuições, e a possível extração e reutilização de artefatos na concepção de novos produtos de software a partir da análise dos softwares e aplicações considerados.

O artigo está organizado da forma que segue: a Seção 2 apresenta a metodologia, planejamento e execução do mapeamentos sistemático, bem como os principais resultados obtidos. A Seção 3 apresenta as discussões das questões de pesquisa. A Seção 4 apresenta as lacunas identificadas e a Seção 5 apresenta as conclusões e indicações de trabalhos futuros.

\section{Mapeamento Sistemático: Metodologia, Planejamento e Execução}

O mapeamento sistemático conduzido neste estudo seguiu as diretrizes propostas por [Kitchenham and Charters 2007] e por [Biolchini et al. 2005]. Cinco questões de pesquisa (QP) foram definidas para serem respondidas com base nos estudos primários recuperados:

QP1. Quais softwares educacionais têm apoiado o ensino de programação?

QP2. Quais as modalidades de ensino apoiadas?

QP3. Qual a linguagem de programação, sistema operacional (SO) e técnicas de desenvolvimento são adotadas?

QP4. Quais as estratégias de ensino adotadas? 
QP5. De que forma os softwares e aplicações educacionais foram avaliados?

\subsection{Busca e Seleção}

As diversas atividades conduzidas para a busca, seleção dos estudos e para a realização da extração dos dados ${ }^{1}$ são apresentadas na Figura 1.

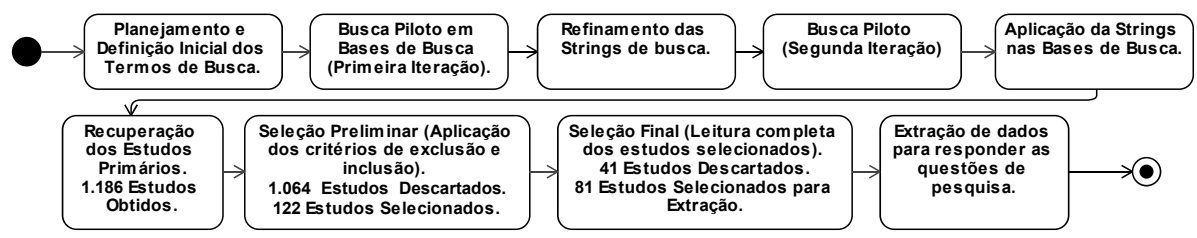

Figura 1. Atividades do Processo de Busca e Seleção de Estudos Primários.

A primeira atividade (Figura 1) corresponde ao planejamento, no qual foram definidas as questões. Em seguida o protocolo PICOC (population, intervention, comparison, outcome, context) [Petticrew and Roberts 2006] permitiu a definição, juntamente com duas iterações piloto, dos termos de busca: "software educacional" e "ensino de programação" e "educational software" e "programming teaching", com seus sinônimos e variações.

Os termos de busca definidos foram aplicados nas bibliotecas digitais e bases indexadas da ACM, IEEE, ScienceDirect, Compendex e Scopus (em inglês). Também foram consideradas as bases da Revista Brasileira de Informática na Educação (RBIE) e de periódicos da Coordenação de Aperfeiçoamento de Pessoal de Nível Superior (CAPES) (em português). A busca foi realizada em 2014, abrangendo o período de 2004 a 2014.

$\mathrm{Na}$ atividade de seleção preliminar foram aplicados critérios de inclusão e exclusão. O critério de inclusão restringe a seleção aos estudos que abordam softwares ou aplicações educacionais para o ensino de programação e conteúdos relacionados. Já os critérios de exclusão foram: estudos primários resumidos, estudos duplicados, estudos indisponíveis e estudos em outros idiomas que não sejam o inglês e o português. Ao final da seleção preliminar foram obtidos 122 estudos.

Os 122 estudos primários selecionados foram submetidos à leitura na íntegra, sendo que 81 foram selecionados para responder as questões de busca definidas. A Tabela 1 sumariza os resultados obtidos nas etapas de seleção.

Tabela 1. Estudos Primários Recuperados por Base de Busca.

\begin{tabular}{l|l|l|l|l|l|l}
\hline$\#$ & Base & Qtd. & $\begin{array}{c}\text { Descartados } \\
\text { (Seleção Preliminar) }\end{array}$ & $\begin{array}{c}\text { Seleção } \\
\text { Preliminar }\end{array}$ & $\begin{array}{c}\text { Descartados } \\
\text { (Seleção Final) }\end{array}$ & Seleção Final \\
\hline 1 & ACM & 38 & 35 & 3 & 2 & 1 \\
2 & Compendex & 397 & 325 & 72 & 20 & 52 \\
3 & IEEE & 40 & 29 & 11 & 3 & 8 \\
4 & Periódicos CAPES & 63 & 62 & 1 & 1 & 0 \\
5 & RBIE & 10 & 9 & 1 & 0 & 1 \\
6 & ScienceDirect & 17 & 11 & 6 & 2 & 4 \\
7 & Scopus & 621 & 593 & 28 & 13 & 15 \\
\hline & Total & $\mathbf{1 1 8 6}$ & $\mathbf{1 0 6 4}$ & $\mathbf{1 2 2}$ & $\mathbf{4 1}$ & $\mathbf{8 1}$ \\
\hline
\end{tabular}

Os estudos recuperados apresentaram maior concentração de publicação nos anos de 2012 a 2013, como observado no gráfico da Figura 2.

\footnotetext{
${ }^{1} \mathrm{O}$ protocolo completo e as referências podem ser consultados em https://goo.gl/4hulgz
} 


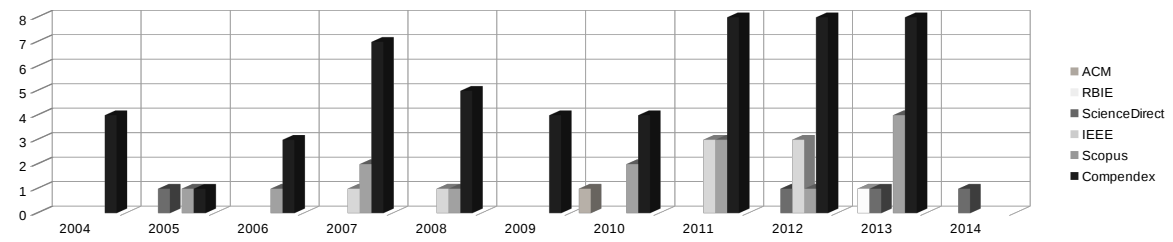

Figura 2. Quantidade de Estudos por Ano para cada Base de Busca.

Em relação aos conteúdos de programação abordados, 24 estudos (e seus respectivos softwares e aplicações) referem-se ao ensino da linguagem Java, 17 abordam fundamentos de programação, oito estudos C, sete estudos orientação a objetos, dois abordam estrutura de dados e algoritmos, dois linguagem LOGO, 12 estudos abordam linguagens de programação específicas (tais como C\#, Python, ASP.Net, C++, etc) e nove não descrevem precisamente. É importante mencionar que o conteúdo de fundamentos de programação foi adotado inclusive em níveis escolares elementares.

\section{Discussão das Questões de Pesquisa}

A seguir são apresentadas as respostas para as questões de pesquisa do mapeamento.

QP1. Quais softwares educacionais têm apoiado o ensino de programação?

A Tabela 2 apresenta o catálogo dos 81 estudos e o nome dos respectivos softwares e aplicações identificados (coluna "Software") para promover o ensino de programação. Mais detalhes são apresentados nas respostas às demais questões de pesquisa.

\section{QP2. Quais as modalidades de ensino apoiadas?}

A Tabela 3 (a) apresenta as modalidades de ensino consideradas nos estudos primários recuperados. Os estudos que não especificaram explicitamente a modalidade, foram classificados de acordo com suas características.

Dos 81 estudos, 35 são utilizados isoladamente, em desktops, 30 referemse a ambientes de aprendizagem eletrônicos (e-learning) [Georgieva et al. 2005], dois estudos abordam tecnologias móveis (m-learning) e apenas um estudo aborda tanto m-learning quanto e-learning, sendo classificado como blended learning. Constatase ainda a inexistência de estudos que utilizam a modalidade de ensino t-learning [Monteiro et al. 2010].

\section{QP3. Qual a linguagem de programação, sistema operacional (SO) e técnicas de desenvolvimento são adotadas?}

As Tabelas 3 (b), (c) e (d) apresentam as linguagens de programação, os sistemas operacionais (SOs) e as tecnologias de desenvolvimento adotadas nos softwares e aplicações, respectivamente.

A linguagem de programação mais utilizada no desenvolvimento dos softwares e aplicações foi a linguagem Java (24 estudos), o que os caracteriza como softwares e aplicações independentes de plataforma, pela tecnologia da máquina virtual Java. A Tabela 3 (c) reflete esta constatação, visto que 35 são multiplataforma. Já em relação às técnicas, abordagens e tecnologias adotadas, 21 utilizam tecnologias específicas para o desenvolvimento, tais como o uso do Google App Engine, a ferramenta Hot-Potato, Java 
CBIE-LACLO 2015

Anais do XXVI Simpósio Brasileiro de Informática na Educação (SBIE 2015)

Tabela 2. Catálogo de Softwares e Aplicações Educacionais para o Ensino de Programação

\begin{tabular}{|c|c|c|c|c|c|c|c|}
\hline Id & $\begin{array}{c}\text { Tipo de } \\
\text { Publicação }\end{array}$ & Software & Ano & Id & \begin{tabular}{|c|} 
Tipo de \\
Publicação
\end{tabular} & Software & Ano \\
\hline E1 & Conference & Jype & 2010 & E42 & Conference & $\begin{array}{l}\text { Generic Code-Memory Diagram } \\
\text { Animation Creator }\end{array}$ & 2004 \\
\hline E2 & Conference & JFLAP & 2013 & $\mathrm{E} 43$ & Conference & PGT & 2007 \\
\hline E3 & Conference & Cloud C++ Workbook & 2013 & E44 & Conference & Greenfoot & 2004 \\
\hline E4 & Journal & MoCAS & 2014 & $\mathrm{E} 45$ & Conference & TouchDevelop & 2012 \\
\hline E5 & Workshop & Eclipse com Eli & 2005 & E46 & Conference & BlueFix & 2012 \\
\hline E6 & Journal & COALA & 2005 & E47 & Conference & $\begin{array}{l}\text { Multi-faceted Support for } \\
\text { MOOC }\end{array}$ & 2012 \\
\hline E7 & Conference & Dolittle & 2007 & $\mathrm{E} 48$ & Conference & ViLLE & 2010 \\
\hline E8 & Conference & $\begin{array}{l}\text { Graphical Tutoring of } \\
\text { Message Passing }\end{array}$ & 2008 & E49 & Conference & FLOP & 2012 \\
\hline E9 & Conference & PlayLOGO 3D & 2011 & $\mathrm{E} 50$ & Conference & Web-Based Environment & 2005 \\
\hline E10 & Conference & $\begin{array}{l}\text { A Framework for Programming } \\
\text { Process Measurement }\end{array}$ & 2011 & E51 & Conference & NXT-G & 2013 \\
\hline E11 & Conference & $\begin{array}{l}\text { Java Programming } \\
\text { Learning System }\end{array}$ & 2012 & E52 & Conference & PROGTEST & 2011 \\
\hline E12 & Conference & TSUNA-TASTE & 2011 & E53 & Conference & SimplifIDE & 2008 \\
\hline E13 & Conference & $\begin{array}{l}\text { Eclipse with } \\
\text { Robot Simulator }\end{array}$ & 2012 & E54 & Conference & BeReT & 2006 \\
\hline E14 & Conference & $\begin{array}{l}\text { HIT Typing, Serious Cube and } \\
\text { Two 3D games }\end{array}$ & 2012 & E55 & Conference & $\begin{array}{l}\text { PROgram Visualization } \\
\text { Tool }\end{array}$ & 2009 \\
\hline E15 & Conference & CABLE & 2006 & E56 & Transactions & Greenfoot & 2010 \\
\hline E16 & Transactions & $\begin{array}{l}\text { Alice, Greenfoot and } \\
\text { Scratch }\end{array}$ & 2010 & E57 & Conference & MUPPETS & 2004 \\
\hline E17 & Conference & AgentWeb & 2012 & E58 & Conference & CodeSpells & 2013 \\
\hline E18 & Conference & $\begin{array}{l}\text { Tool for web-based } \\
\text { animations }\end{array}$ & 2005 & E59 & Conference & Storytelling Alice & 2007 \\
\hline E19 & Conference & DrRacket & 2011 & E60 & Conference & KU-UZEM & 2010 \\
\hline E20 & Conference & CAL-CT Tutors & 2011 & E61 & Conference & $\begin{array}{l}\text { Learning Environment for } \\
\text { Algorithm and Programming }\end{array}$ & 2009 \\
\hline E21 & Conference & VIP Visualization Tool & 2007 & E62 & Transactions & ALVIS Live & 2006 \\
\hline E22 & Journal & WebHat & 2013 & E63 & Conference & mJeliot & 2011 \\
\hline E23 & Conference & LDS & 2010 & E64 & Journal & AutoLEP & 2004 \\
\hline E24 & Conference & ANNA & 2008 & E65 & Conference & $\begin{array}{l}\text { Learning Environment for } \\
\text { Understanding of Program } \\
\text { Division Patterns }\end{array}$ & 2007 \\
\hline E25 & Journal & UOM-TMD & 2011 & E66 & Transactions & State-Based Squeak & 2012 \\
\hline E26 & Conference & C-Sheep & 2007 & E67 & Conference & PROGTEST & 2008 \\
\hline E27 & Conference & $\begin{array}{l}\text { Modkit and LilyPad } \\
\text { ProtoSnap board }\end{array}$ & 2013 & E68 & Conference & RPA System for MOODLE & 2011 \\
\hline E28 & Conference & $\begin{array}{l}\text { Automatically Generated } \\
\text { Programming Tutorials }\end{array}$ & 2013 & E69 & Transactions & $\begin{array}{l}\text { Peer-Learning Agent } \\
\text { Based on Pair Programming }\end{array}$ & 2010 \\
\hline E29 & Journal & JPLAS & 2013 & E70 & Conference & $\begin{array}{l}\text { Tool Programming } \\
\text { Process Visualizer }\end{array}$ & 2013 \\
\hline E30 & Conference & Pick Cricket & 2011 & E71 & Conference & $\begin{array}{l}\text { Real-time Monitoring System for } \\
\text { Programming Education }\end{array}$ & 2006 \\
\hline E31 & Conference & SICAS & 2007 & E72 & Conference & MDAT & 2013 \\
\hline E32 & Journal & Web-CAT & 2008 & E73 & Transactions & Jeliot ConAn & 2010 \\
\hline E33 & Conference & Tutoring System & 2008 & E74 & Journal & COALA & 2012 \\
\hline E34 & Conference & $\begin{array}{l}\text { Active-learning } \\
\text { Environment }\end{array}$ & 2011 & E75 & Conference & ASIDE & 2013 \\
\hline E35 & Conference & MOOC & 2013 & E76 & Conference & $\begin{array}{l}\text { Game-Based Concept } \\
\text { Visualization for Learning Programming }\end{array}$ & 2011 \\
\hline E36 & Workshop & Prolog Visualization System & 2009 & E77 & Conference & JPL & 2013 \\
\hline E37 & Conference & $\begin{array}{l}\text { Corrective Feedback and Concept } \\
\text { Visualisation Tool }\end{array}$ & 2011 & E78 & Journal & QA cycle execution system & 2007 \\
\hline E38 & Conference & Parson's Programming Puzzles & 2006 & E79 & Conference & 3DAPS & 2011 \\
\hline E39 & Workshop & Jason (AgentSpeak(L)) & 2012 & E80 & Conference & JBuilder & 2012 \\
\hline $\mathrm{E} 40$ & Conference & MSWLogo & 2007 & E81 & Conference & $\begin{array}{l}\text { LEGO Mindstorms } \\
\text { Simulator (LMS) }\end{array}$ & 2008 \\
\hline E41 & Conference & $\begin{array}{l}\text { Pen-Based Flowchart } \\
\text { Recognition System }\end{array}$ & 2008 & & & & \\
\hline
\end{tabular}

Applet, protocolo de comunicação baseado em XML, etc. Os poucos estudos que apresentam indicações de reuso estão relacionadas à tecnologia Java (7), a utilização de Modelview-controller (2), arquiteturas orientadas a serviços (3) e desenvolvimento baseado em componentes (1). 
CBIE-LACLO 2015

Anais do XXVI Simpósio Brasileiro de Informática na Educação (SBIE 2015)

Tabela 3. Especificações Técnicas.

\begin{tabular}{|c|c|c|c|c|c|}
\hline \# & \multicolumn{4}{|c|}{ Modalidade } & Qtd. \\
\hline 1 & \multicolumn{4}{|c|}{ Ensino presencial mediado por tecnologias } & 35 \\
\hline 2 & \multicolumn{4}{|c|}{ Eletronic learning (e-learning) } & 30 \\
\hline 3 & \multicolumn{4}{|l|}{ Não especificado } & 5 \\
\hline 4 & \multicolumn{4}{|c|}{ Ensino presencial mediado com Jogos /Gameficação ou Design de Jogos } & 4 \\
\hline 5 & \multicolumn{4}{|c|}{ Ensino presencial mediado por Robôs/ProtoSnap Board } & 3 \\
\hline 6 & \multicolumn{4}{|c|}{ Blended learning (B-learning) } & 2 \\
\hline 7 & \multicolumn{4}{|c|}{ Mobile Learning (M-learning) } & 2 \\
\hline & \multicolumn{4}{|c|}{ Total } & 81 \\
\hline \multicolumn{3}{|c|}{ b) Linguagens de Programação } & \multicolumn{3}{|c|}{ c) Tecnologias de Desenvolvimento } \\
\hline \# & Linguagem & Qtd. & \# & Tecnologias de Desenvolvimento & Qtd. \\
\hline 1 & Não especificado & 47 & 1 & Não especificado & 60 \\
\hline 2 & Java & 24 & 2 & Tecnologia Java & 7 \\
\hline 3 & Outras Linguagens & 6 & 3 & Arquitetura Model-View-Controller (MVC) & 2 \\
\hline 4 & C\#/ASP.Net & 4 & 4 & Arquitetura orientada a serviços & 3 \\
\hline \multicolumn{2}{|r|}{ Total } & 81 & 5 & Conjunto de instruções RISC (linguagem de máquina) & 1 \\
\hline \multicolumn{3}{|c|}{ d) Sistema Operacional/Plataforma } & 6 & Google App Engine & 1 \\
\hline \# & SO/Plataforma & Qtd. & 7 & Abordagem baseada em componentes & 1 \\
\hline 1 & Multiplataforma & 35 & 8 & Módulo de fisica Open Dynamics Engine & 1 \\
\hline 2 & Não especificado & 34 & 9 & Integração com camadas de middleware multi agente & 1 \\
\hline 3 & Windows & 5 & 10 & Cliente móvel, servidor e protocolo de comunicação baseado em XML & 1 \\
\hline 4 & Linux e Windows & 2 & 11 & Desenviolamento com Hot-Potato & 1 \\
\hline 5 & Windows Phone & 1 & 12 & XML para troca de mensagens (servidor e módulo) & 1 \\
\hline 6 & Mac OS X & 1 & 13 & Arquitetura de terceira geração, com armazenador de logs integrado. & 1 \\
\hline 7 & Windows e iOS & 1 & \multicolumn{2}{|r|}{ Total } & 81 \\
\hline 8 & Android & 1 & & & \\
\hline 9 & UNIX & 1 & & & \\
\hline & Total & 81 & & & \\
\hline
\end{tabular}

Ao identificar os estudos que utilizam padrões para seu desenvolvimento sejam dos softwares e aplicações ou de objetos de aprendizagem e outros elementos, identificouse que apenas 1 estudo adota o padrão Shareable Content Object Reference Model $(\mathrm{SCORM})^{2}$ - KU-UZEM [E60].

\section{QP4. Quais as estratégias de ensino adotadas?}

A pesquisa na literatura sobre o ensino de fundamentos de programação, discutindo principalmente os currículos de cursos de programação [Pears et al. 2007], apresenta uma classificação para softwares e aplicações educacionais, segundo as ferramentas que usam estratégias distintas. Esta classificação resulta em 5 tipos de ferramentas de software: (i) ferramentas de visualização; (ii) de avaliação automática; (iii) de apoio à programação; (iv) micromundos; e (v) outras ferramentas, que usam estratégias específicas, não incluídas nas categorias anteriores. Com base nesses tipos de ferramentas e suas estratégias, os softwares e aplicações identificados nos estudos foram classificados.

A Figura 3 apresenta o buble plot das estratégias em relação ao ano em que foram publicadas e ao total de estudos por categoria. Nota-se uma diversificação nas estratégias adotadas. Algumas delas integram conceitos pedagógicos interessantes para motivar e instruir os alunos, como o CABLE (Cognitive Apprenticeship-Based Learning Environment) [E15], que fornece retorno ao instrutor por meio de avaliação cognitiva da aprendizagem do aluno. Já o SimplifIDE [E53] propõe um IDE simplificado para facilitar o entendimento de conceitos relacionados à programação.

Alguns estudos abordam o ensino de programação para níveis básicos de escolari-

\footnotetext{
${ }^{2}$ http: / / www. scormsoft.com/scorm
} 


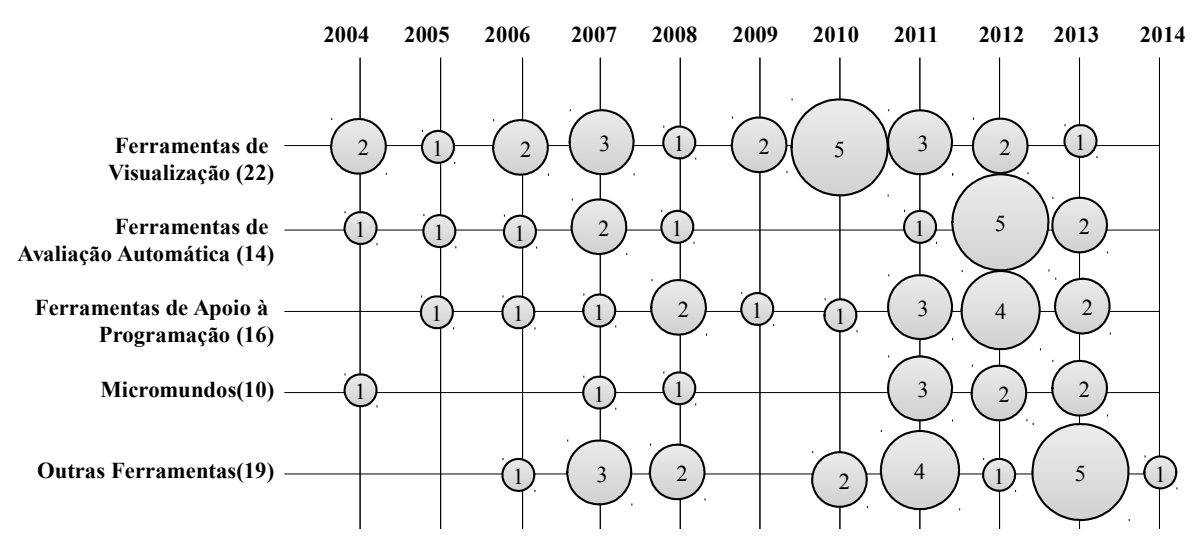

Figura 3. Estratégias de Software e Ano de Desenvolvimento.

dade, como em [E16], que apresenta três ferramentas que apoiam o ensino em diferentes fases: Alice para estudantes de nível primário; Scratch, para os de idade de 8 a 16 anos e o Greenfoot, para os com idade superior a 14 anos. As três integram conceitos visuais e lúdicos para promover e motivar os estudantes.

Entre estratégias de visualização de código, destacam-se as que apresentam o código por meio de blocos de montar, permitindo interação do aluno com avatar 3D (3D Avatar Programming System) [E79], e as que adotam conceitos lúdicos. A utilização de estratégias de visualização apoia o aprendizado, facilitando-o, principalmente, para os conceitos básicos de programação para alunos de séries elementares. Promove-se também a motivação, uma vez que a diversão é a intermediadora entre aluno e conteúdos ensinados.

Alguns estudos também adotam a interatividade, como o uso de tablets e controles de vídeo game, para motivar discentes de diferentes níveis escolares, como quatro jogos apresentados no estudo [E14]. Ainda há iniciativas que promovem a criação de animações 3D, Storytelling Alice [E59], adaptação da ferramenta Alice [E16] e as que utilizam metáforas, representando comandos e funções em "feitiços" - CodeSpells [E58]. O uso de metáforas é classificado como micromundos, termo que designa ambientes de aprendizagem nos quais é possível explorar, descobrir e simular acontecimentos da vida real ou de fantasia [JONASSEN 2007].

A criação de plugins com ambientes de desenvolvimento integrado (IDE) como em [E5, E6, E13, E21, E75] também é apresentada como estratégia, com destaque para o COALA (Computer Assisted Environment for Learning Algorithms) [E6]. Ele integra plugins para a IDE Eclipse, criando um ambiente web integrado. Existem, ainda, as ferramentas de apoio à programação, e as que apoiam a avaliação automatizada, as que utilizam a web, acesso remoto, armazenamento na nuvem, e outras tecnologias de comunicação, como dispositivos móveis [E11, E12, E20, E22, E28, E71, E77]. Algumas propostas englobam agentes inteligentes para fornecer retorno, tanto ao corpo discente como docente, auxiliam as atividades de desenvolvimento e apontam erros cometidos durante as atividades [E67, E68].

Há, por fim, estratégias diferenciadas, classificadas como "outras ferramentas", como as que utilizam robôs, tanto reais quanto virtuais, para permitir a visualização da 
interação do código com os mesmos [E27, E30, E34, E51, E81]. Destaca-se, também, as que utilizam hardware especial, como o Modkit e LilyPad ProtoSnap board [E27], que permitem a programação de proto boards costuráveis em roupas para motivar, sobretudo, estudantes do sexo feminino. Outra estratégia que se destaca no monitoramento do desempenho dos alunos e os reporta ao professor, visando que esse possa modificar as estratégias de aula adotadas, é a chamada Real-time Monitoring System for Programming Education [E71].

\section{QP5. De que forma os softwares e aplicações educacionais foram avaliados?}

Dos 81 estudos, 24 aplicaram questionários aos usuários para avaliar o uso do software/aplicação. Por meio destes foram identificados problemas e o quão aptas tais iniciativas estavam, nas perspectivas dos alunos e professores, para ensinar o conteúdo de programação abordado.

Dos demais estudos, 24 avaliaram resultados coletados nos softwares, como notas e pontuações atribuídas na realização de atividade. Apenas 11 estudos adotaram formalismo experimental, permitindo o teste de hipóteses, por meio de comparações com outros recursos instrucionais para a aprendizagem de programação. Quatro estudos aplicaram comparação dos resultados entre softwares, técnicas e grupos de controle porém, sem o apoio de técnicas estatísticas. Seis estudos utilizaram técnicas de avaliação específicas, como comparações qualitativas, estudos de caso, hipóteses testadas por questionário, análise do conhecimento pré e pós uso do software. Finalmente, 12 estudos não apresentam avaliações, dentre os quais, alguns autores indicam a possível realização destas em estudos futuros. No entanto, apenas um trabalho apresentou continuidade na pesquisa, realizando a avaliação indicada previamente [E67, E52].

\section{Lacunas Encontradas}

A partir do mapeamento sistemático conduzido, é possível observar que as propostas de softwares e aplicações educacionais para o ensino de programação apontam diversas questões e também possibilidades a serem exploradas, conforme elencado a seguir:

Tópicos abordados: a predominância de softwares e aplicações que abordam o ensino de programação com linguagens específicas, como a linguagem Java e C, deve ser avaliada, visto que se os fundamentos de programação não estão claramente entendidos pelos estudantes, a utilização de uma linguagem específica pode não resultar no atendimento completo do objetivo educacional almejado, ou seja, aprender a programar.

Modalidades de Ensino: a ainda incipiente exploração de tecnologias, como dispositivos móveis e televisores, e a restrição no uso de tecnologias apenas nos ambientes escolares, motivam uma adoção mais efetiva das modalidades de m-learning e t-learning. Tais modalidades devem ser avaliadas quanto ao seu uso como mediadoras para o ensino de programação em diversos níveis escolares, pervasivas à rotina dos alunos.

Especificações Técnicas: As linguagens de programação, SOs/plataformas e as técnicas adotadas para o desenvolvimento das propostas são aplicadas, essencialmente, no método singular de desenvolvimento. Não foram observados esforços significativos no intuito de facilitar o processo de reuso e nenhuma delas utiliza uma metodologia de reutilização sistemática, como linhas de produto de software (LPS).

O uso de LPSs, em especial, garante o reuso de diferentes artefatos [Krueger 2002] 
e permite o desenvolvimento de produtos de software mais adaptados às especificidades de aprendizagem dos alunos e exigências dos currículos e docentes. Além disso, a não adoção de reutilização limita a evolução e inclusão de novas funcionalidades nos produtos existentes, exigindo maiores esforços e recursos. Além disso, a falta de padrões de desenvolvimento, assim como a não disponibilização do código aberto de alguns dos produtos apresentados, prejudica o reuso destes.

Avaliações Experimentais: As poucas avaliações experimentais levam a questionamentos quanto aos estudos que utilizam apenas resultados obtidos por retorno dos usuários, uma vez que podem não evidenciar os reais resultados, se submetidos a testes estatísticos.

A concepção isolada dos softwares e aplicações, por sua vez, sem a integração de diferentes grupos de pesquisa e, também, com pouca abrangência em relação aos problemas de programação que enfrentam, restringem a sua adoção em instituições diferentes das quais foram desenvolvidos. A execução de replicações experimentais, por exemplo, em outros grupos de pesquisa, poderia tanto ajudar a identificar se a adoção destes softwares/aplicações é válida para outras instituições quanto verificar o quão adaptáveis e amplas são as soluções em relação aos problemas de ensino e aprendizagem de programação.

Técnicas e Estratégias para Promover o Ensino de Programação: foi observado que as principais estratégias aplicam conceitos visuais, elementos lúdicos e outras utilizam a Internet para garantir que as atividades possam ter continuidade fora dos limites escolares. Porém, em conjunto com as modalidades de ensino e também especificações técnicas, a utilização de outros elementos tecnológicos, tais como as tecnologias móveis e televisivas, ainda são pouco explorados. Estas tecnologias, amplamente difundidas [CGI 2014], tornam-se meios em potencial de atingir um maior número de aprendizes e consequentemente, um meio de motivá-los a aprender a programar. Portanto, identificar se tais estratégias são válidas e se podem expandir as fronteiras da aprendizagem de programação ao serem integradas a modalidades de ensino a distância, é uma lacuna interessante a ser estudada.

\section{Conclusões e Trabalhos Futuros}

O mapeamento sistemático apresentado neste artigo identificou as principais contribuições de software para promover o ensino de programação e tópicos relacionados. Os 81 estudos primários recuperados contribuíram para formar uma base de referencial teórico expressando a realidade em relação às especificações técnicas, avaliações experimentais e estratégias para a disseminação e aprendizagem de programação.

A classificação e análise destes estudos em relação às suas características, formando um catálogo de contribuições de softwares e aplicações, permite uma visão mais precisa dos mesmos, para uma possível adoção. Além disso, foi possível identificar as principais lacunas a serem tratadas e inferir, principalmente, que há a falta de sinergia entre as diferentes iniciativas e instituições, na busca por soluções integradas, adaptáveis às exigências educacionais dos discentes e docentes e, com soluções que abranjam uma maior gama de problemas enfrentados.

Como trabalhos futuros pretende-se explorar as modalidades de ensino m-learning e $t$-learning, em conjunto com diferentes estratégias de ensino de programação. Para isso, 
técnicas de desenvolvimento que propiciem a evolução facilitada devem ser investigadas, permitindo instanciar e avaliar softwares e aplicações educacionais especificamente voltados ao ensino e aprendizagem de programação, em diferentes níveis escolares.

\section{Agradecimentos}

Os autores agradecem o apoio da Coordenação de Aperfeiçoamento de Pessoal de Nível Superior (CAPES) (DS-8907173DT).

\section{Referências}

Aureliano, V. C. O. and Tedesco, P. C. A. R. (2012). Ensino e Aprendizagem de Programação para Iniciantes: uma Revisão Sistemática da Literatura focada no SBIE e WIE. Simp. Brasileiro de Inf. na Educação.

Biolchini, J., Mian, P. G., and Natali, A. C. C. (2005). Systematic Review in Software Engineering. Technical Report RT-ES 679/05, COPPE/UFRJ, Rio de Janeiro, RJ, Brasil.

CGI (2014). Comitê Gestor da Internet no Brasil. Pesquisa Sobre o Uso das Tecnologias de Informação e Comunicação no Brasil: TIC Domícilios e Empresas 2013. São Paulo.

Georgieva, E., Smrikarov, A., and Georgiev, T. (2005). A General Classification of Mobile Learning Systems. In International conference on computer systems and technologies CompSysTech, volume 8.

IEEE and ACM (2005). IEEE Computer Society Press and ACM Press. Joint Task Force for Computing Curricula. Disponível em http: / / www1. acm. org/education/curricula. html Último acesso em: 03 Jul. 2014.

JONASSEN, D. (2007). Computadores x ferramentas cognitivas: Desenvolver o Pensamento Crítico nas Escolas. Porto.

Kitchenham, B. and Charters, S. (2007). Guidelines for Performing Systematic Literature Reviews in Software Engineering. Technical Report EBSE 2007-001, Keele University.

Krueger, C. W. (2002). Easing the Transition to Software Mass Customization. In 4th Int. Workshop on Software Product-Family Engineering, PFE '01, pages 282-293, London, UK. Springer-Verlag.

Monteiro, B., Prota, T., Gomes, A., and de Souza, F. (2010). Amadeus-tv: Portal educacional na tv digital integrado a um sistema de gestão de aprendizado. Revista Brasileira de Inf. na Educação, 18(01).

Mota, M. P., de Brito, S. R., Moreira, M. P., and Favero, E. L. (2009). Ambiente Integrado à Plataforma Moodle para Apoio ao Desenvolvimento das Habilidades Iniciais de Programação. Simp. Brasileiro de Inf. na Educação.

Pears, A., Seidman, S., Malmi, L., Mannila, L., Adams, E., Bennedsen, J., Devlin, M., and Paterson, J. (2007). A survey of literature on the teaching of introductory programming. SIGCSE Bull., 39:204-223.

Petticrew, M. and Roberts, H. (2006). Systematic Reviews in the Social Sciences: A Practical Guide. Blackwell Publishing.

S., A. W., M., R. B., A., N. E., F., F. R., and de Melo Nunes Soares F. A. A. (2012). RHODES 2.0: Software Educacional para o Ensino de Programação Linear. Simpósio Brasileiro de Informática na Educação.

Sales, C. G. and Dantas, V. F. (2010). ProGame: um Jogo para o Ensino de Algoritmos e Programação. Simpósio Brasileiro de Informática na Educação.

SBC (2003). Sociedade Brasileira de Computação. Currículo de Referência da Sociedade Brasileira de Computação para Cursos de Graduação em Computação e Informática. Disponível em http: / / www . sbc . org . br / Último acesso em: 17 de Dez. de 2014. 